\title{
COUPLED POINTS IN THE CALCULUS OF VARIATIONS AND APPLICATIONS TO PERIODIC PROBLEMS
}

\author{
VERA ZEIDAN AND PIERLUIGI ZEZZA
}

\begin{abstract}
The aim of this paper is to introduce the definition of coupled points for the problems of the calculus of variations with general boundary conditions, and to develop second order necessary conditions for optimality. When one of the end points is fixed, our necessary conditions reduce to the known ones involving conjugate points. We also apply our results to the periodic problems of the calculus of variations.
\end{abstract}

\section{INTRODUCTION AND PRELIMINARY RESULTS}

In this paper we will consider the following boundary value problem in the calculus of variations, denoted by (BVP):

Minimize

$$
J(x):=\gamma(x(a), x(b))+\int_{a}^{b} L(s, x(s), \dot{x}(s)) d s
$$

over all absolutely continuous functions $x(\cdot)$, subject to the boundary condition

$$
\delta(x(a), x(b))=0,
$$

where $\gamma: \mathbf{R}^{n} \times \mathbf{R}^{n} \rightarrow \mathbf{R}, \delta: \mathbf{R}^{n} \times \mathbf{R}^{n} \rightarrow \mathbf{R}^{k},(k \leq 2 n)$, and $L:[a, b] \times \mathbf{R}^{n} \times \mathbf{R}^{n} \rightarrow$ R.

The fact that the boundary condition (1.2) is expressed by a function of both $x(a)$ and $x(b)$ gives the problem a general aspect. It encompasses the case where $x(a)$ and $x(b)$ are restricted to belong to two manifolds, as well as the case where the boundary conditions are periodic.

This problem has been a subject of study since its formulation in the 17 th century. One can find an extensive literature on this question concerning second order necessary conditions. In this context, for the special case of fixed endpoints, the theory of conjugate points was developed and analyzed in depth, see,

Received by the editors March 21, 1988.

1980 Mathematics Subject Classification (1985 Revision). Primary 49B10.

Key words and phrases. Calculus of variations, nonlinear boundary value problems, coupled points, periodic problems.

The authors wish to thank G.N.A.F.A. of C.N.R. (Italy) and N.S.E.R.C. (Canada) whose financial support made this research possible.

Research of the second author partly supported by research grant M.P.I. 40\%, Teoria del Controllo dei Sistemi Dinamici. 
e.g., [3], where it is given in terms of envelopes as well as in terms of properties of the solutions of a second order selfadjoint differential equation.

Several attempts were made to extend this theory to more general boundary conditions. For instance, in 1898, Kneser studied this question for the case of one variable endpoint and in 1903, Bliss developed a theory for two-variable endpoints in $\mathbf{R}^{2}$ based on the special properties of the plane so that it does not extend to a higher dimension; in this context, conjugate points carried the name of focal points. Both contributions were originally expressed in terms of envelopes of extremals. These results, together with the contributions of a very active group of mathematicians who worked at the University of Chicago around 1930, are presented in [2], where an analytical definition of a conjugate point and a focal point (with one variable end point) is provided. A modern presentation of these results can be found in [8].

Since the development of the optimal control theory, the interest in studying general boundary conditions, and in particular the periodic ones, has increased (see, e.g., [9]). But, as it is apparent, the Jacobi theory for this general type of conditions needs more study even in the simpler case of the calculus of variations.

The main purpose of this paper is to complete the study of second order necessary conditions for the problem of the calculus of variations (BVP). We focus our attention on establishing necessary conditions for the nonnegativity of the second variation. Thus our approach is analytical and does not require the construction of a field of extremals.

In $\S 2$ we introduce the definition of coupled points and the notion of regularity. In the classical setting these concepts reduce to the known definition of conjugate points. We show that both regularity and the nonexistence of coupled points to $b$ in $(a, b)$ are necessary conditions for optimality in (BVP) and thus we generalize the known necessary conditions involving either conjugate or focal points.

In $\S 3$ we apply these results to obtain necessary conditions for the periodic problems of the calculus of variations.

Given a $C^{1}$-function $\hat{x}(\cdot):[a, b] \rightarrow \mathbf{R}^{n}$, an $\varepsilon$-tube around $\hat{x}(\cdot)$ and $\dot{\hat{x}}(\cdot)$ is $T(\hat{x}, \varepsilon)=\left\{(u, v) \in \mathbf{R}^{n} \times \mathbf{R}^{n}:\|u-\hat{x}(t)\|<\varepsilon,\|v-\dot{\hat{x}}(t)\|<\varepsilon\right.$ for some $\left.t \in|a, b|\right\}$.

A Lipschitz function $\hat{x}:|a, b| \rightarrow \mathbf{R}^{n}$ that satisfies (1.2) is a weak local minimum for (BVP) if $J(x) \geq J(\hat{x})$ for all admissible $x(\cdot)$ in a weak neighbourhood of $\hat{x}(\cdot)$, that is, a neighbourhood in $H^{1, \infty}$.

The following regularity assumptions on the data, which we adopt, are usually made while studying the classical Jacobi necessary condition in the basic problem of the calculus of variations.

The problem (BVP) has a weak local minimum at $\hat{x} \in C^{1}\left([a, b], \mathbf{R}^{n}\right)$; the function $L$ is $C^{2}$ on $[a, b] \times T(\hat{x}, \varepsilon)$, where $T(\hat{x}, \varepsilon)$ is an $\varepsilon$-tube around $\hat{x}(\cdot)$ and $\dot{\hat{x}}(\cdot)$, and the functions $\gamma, \delta$ are $C^{2}$ in a neighbourhood of $(\hat{x}(a), \hat{x}(b))$. 
Moreover, we assume that the differential of $\delta(\cdot)$ at $(\hat{x}(a), \hat{x}(b))$ is surjective and the strengthened Legendre condition holds along the optimal trajectory:

$$
L_{v v}(t, \hat{x}(t), \dot{\hat{x}}(t))>0 \quad \forall t \in[a, b] .
$$

Under these assumptions the minimizer $\hat{x}(\cdot)$ satisfies the Euler-Lagrange equation

$$
\frac{d}{d t} \widehat{L}_{v}(t)=\widehat{L}_{x}(t), \quad t \in[a, b],
$$

and the following transversality conditions (see, e.g., [3]): there exists $\mu \in \mathbf{R}^{k}$, such that

$$
\begin{gathered}
\widehat{L}_{v}(a)=\gamma_{x}(\hat{x}(a), \hat{x}(b))+\delta_{x}^{T}(\hat{x}(a), \hat{x}(b)) \mu, \\
\widehat{L}_{v}(b)=-\gamma_{y}(\hat{x}(a), \hat{x}(b))-\delta_{y}^{T}(\hat{x}(a), \hat{x}(b)) \mu,
\end{gathered}
$$

where " " denotes the evaluation along the optimal arc $\hat{x}(\cdot)$.

Under these assumptions, consider the accessory boundary value problem $(B V P)^{*}$ associated with (1.1)-(1.2):

Minimize

$$
\begin{aligned}
J_{2}(\eta) & :=\frac{1}{2}\left(\eta(a)^{T}, \eta(b)^{T}\right) \Gamma\left(\begin{array}{c}
\eta(a) \\
\eta(b)
\end{array}\right) \\
& +\frac{1}{2} \int_{a}^{b}\left\{\eta^{T}(s) \widehat{L}_{x x}(s) \eta(s)+2 \dot{\eta}^{T}(s) \widehat{L}_{v x}(s) \eta(s)+\dot{\eta}^{T}(s) \widehat{L}_{v v}(s) \dot{\eta}(s)\right\} d s
\end{aligned}
$$

over all absolutely continuous $\eta(\cdot)$, subject to the boundary conditions

$$
D\left(\begin{array}{l}
\eta(a) \\
\eta(b)
\end{array}\right)=0 \text {, }
$$

where

$$
\begin{gathered}
D:=\nabla \delta(\hat{x}(a), \hat{x}(b)), \\
\Gamma:=\nabla^{2} \gamma(\hat{x}(a), \hat{x}(b))+\sum_{i=1}^{k} \mu_{i} \nabla^{2} \delta_{i}(\hat{x}(a), \hat{x}(b)),
\end{gathered}
$$

and $\mu$ is the same vector as in the transversality conditions. In [5] the following second order necessary condition is proven: the problem (BVP) ${ }^{*}$ has a minimum at $\eta(\cdot) \equiv 0$. That is, for all $\eta \in A C$ satisfying (1.4), the following holds: $J_{2}(\eta) \geq 0$.

In order to simplify the notations we set $P(\cdot)=\widehat{L}_{x x}(\cdot)=\widehat{L}_{v x}(\cdot), R(\cdot)=$ $\widehat{L}_{v v}(\cdot)$. The Euler-Lagrange equation associated with the accessory problem is

$$
\frac{d}{d t}[R(t) \dot{\eta}(t)+Q(t) \eta(t)]=Q^{T}(t) \dot{\eta}(t)+P(t) \eta(t) \quad \text { a.e. } t \in[a, b]
$$

By a solution of this equation we understand a function $\eta(\cdot) \in A C$ such that, for some $\xi(\cdot) \in A C$, we have

$$
\begin{aligned}
& \xi(t)=R(t) \dot{\eta}(t)+Q(t) \eta(t), \\
& \dot{\xi}(t)=Q^{T}(t) \dot{\eta}(t)+P(t) \eta(t) \quad \text { a.e. } t \in[a, b]
\end{aligned}
$$


Whenever $R(\cdot)$ is invertible on $[a, b]$, the above system becomes

$$
\begin{aligned}
& \dot{\eta}(t)=-R^{-1}(t) Q(t) \eta(t)+R^{-1}(t) \xi(t), \\
& \dot{\xi}(t)=Q^{T}(t)\left[-R^{-1}(t) Q(t) \eta(t)+R^{-1}(t) \xi(t)\right]+P(t) \eta(t),
\end{aligned}
$$

or equivalently,

$$
\dot{\eta}(t)=A(t) \eta(t)+B(t) \xi(t), \quad \dot{\xi}(t)=C(t) \eta(t)-A^{T}(t) \xi(t),
$$

where

$$
A(\cdot):=-R^{-1}(\cdot) Q(\cdot), \quad B(\cdot):=R^{-1}(\cdot), \quad C(\cdot):=P(\cdot)-Q^{T}(\cdot) R^{-1}(\cdot) Q(\cdot) .
$$

Equations (1.7) are called the Jacobi system of problem (BVP).

\section{Main Results: Statements AND PROOFS}

In this section, we develop second order necessary conditions for weak local optimality of $\hat{x}(\cdot)$ in (BVP). This goal is accomplished by providing conditions necessary for the nonnegativity of the objective functional $J_{2}(\eta)$ of (BVP) ${ }^{*}$.

We now introduce the definition of coupled points.

Definition 2.1. A point $c \in[a, b)$ is said to be coupled with $b$ if there exists a nonzero $(\eta(\cdot), \xi(\cdot))$, solution of the Jacobi system (1.7) such that

$$
\eta(\cdot) \not \equiv \eta(c) \quad \text { on }[a, c], \quad D\left(\begin{array}{c}
\eta(c) \\
\eta(b)
\end{array}\right)=0
$$

and

$$
\left(\begin{array}{c}
\xi(c) \\
-\xi(b)
\end{array}\right)=\Gamma\left(\begin{array}{c}
\eta(c) \\
\eta(b)
\end{array}\right)+D^{T} \lambda+\left(\begin{array}{c}
\int_{a}^{c} P(s) d s \eta(c) \\
0
\end{array}\right)
$$

for some $\lambda \in \mathbf{R}^{k}$.

Remark 2.1. When the endpoints are fixed, that is, when $D=I_{2 n \times 2 n}$, the last condition in Definition 2.1 can be dropped since $D$ is onto and $\eta(c)=\eta(b)=0$ (from the second condition). Moreover, $\eta(\cdot) \not \equiv \eta(c)=0$ on $[a, c]$ could be eliminated, since otherwise $(\eta(\cdot), \xi(\cdot)) \equiv(0,0)$ on $[a, b]$. Thus, the definition of a coupled point reduces to that of a conjugate point.

By symmetry it is straightforward to define a point $c$ coupled with $a$.

Definition 2.2. A point $c \in[a, b)$ is said to be coupled with $a$ if there exists a nonzero solution $(\eta(\cdot), \xi(\cdot))$ of the Jacobi system (1.7) such that

$$
\eta(\cdot) \not \equiv \eta(c) \quad \text { on }[c, b], \quad D\left(\begin{array}{l}
\eta(a) \\
\eta(c)
\end{array}\right)=0,
$$

and

$$
\left(\begin{array}{c}
\xi(a) \\
-\xi(c)
\end{array}\right)=\Gamma\left(\begin{array}{c}
\eta(a) \\
\eta(c)
\end{array}\right)+D^{T} \lambda+\left(\begin{array}{c}
0 \\
\int_{c}^{b} P(s) d s \eta(c)
\end{array}\right),
$$

for some $\lambda \in \mathbf{R}^{k}$.

The next results generalize to (BVP) the necessary condition of Jacobi that was derived for the fixed endpoint case. 
Theorem 2.1. If $\hat{x}(\cdot)$ is a weak local minimum for $(B V P)$ then there are no points $c \in(a, b)$ coupled with $b$.

Theorem 2.2. If $\hat{x}(\cdot)$ is a weak local minimum for $(B V P)$ then there are no points $c \in(a, b)$ coupled with $a$.

We apply Theorems 2.1 and 2.2 to a variational problem involving a parameter. These two theorems yield the same conclusion concerning this parameter.

Example 1. Let us consider the following family of problems:

Minimize

$$
J(x ; \theta)=\frac{1}{2} x^{2}(\theta)+\frac{1}{2} \int_{0}^{\theta}\left\{\dot{x}^{2}(s)-x^{2}(s)\right\} d s
$$

over all absolutely continuous functions $x(\cdot)$, that satisfy the boundary conditions:

$$
x(0)=0, \quad \text { (the final point } x(\theta) \text { is free }) .
$$

We first note that the particular form of the lagrangian implies that the domain of the functional is indeed $H^{1,2}$. We will restrict $\theta$ to the interval $[0, \pi]$ because it is known [3] that for $\theta>\pi$ the functional $J(x ; \theta)$ has no lower bound. The Jacobi system for the above problem is the harmonic oscillator $\dot{\eta}(t)=\xi(t), \dot{\xi}(t)=-\eta(t)$, whose solutions are of the form $\eta(t)=A \cos t+B \sin t, \xi(t)=-A \sin t+B \cos t$. Since $x(0)$ is fixed, the point $c \in(0, \theta)$ is coupled with $\theta$ if

$$
\left\{\begin{array} { l } 
{ \eta ( c ) = 0 , } \\
{ \xi ( \theta ) = - \eta ( \theta ) }
\end{array} \Leftrightarrow \left\{\begin{array}{l}
A \cos c+B \sin c=0 \\
-A \sin \theta+B \cos \theta+A \cos \theta+B \sin \theta=0 .
\end{array}\right.\right.
$$

Since $\sin c \neq 0$ for $c \in(0, \theta)$, we obtain

$$
\left\{\begin{array}{l}
B=-A \cot c, \\
A(\sin (\theta-c)+\cos (\theta-c))=0 .
\end{array}\right.
$$

We have $A \neq 0$ since otherwise the first relation of (2.1) implies that also $B=0$. The second equation in (2.2) thus yields $\theta-c=\frac{3}{4} \pi$. Since $0<c<\theta$, there are points $c$ coupled with $\theta$ if and only if $\theta>\frac{3}{4} \pi$.

Let us now apply Theorem 2.2. Given that the harmonic oscillator has no nonzero constant solutions, a point $c \in(0, \theta)$ is coupled with 0 if

$$
\left\{\begin{array} { l } 
{ \eta ( 0 ) = 0 } \\
{ \xi ( c ) = \eta ( c ) ( \theta - 1 - c ) }
\end{array} \Leftrightarrow \left\{\begin{array}{l}
A=0 \\
B[\cos c-(\theta-c-1) \sin c]=0 ;
\end{array}\right.\right.
$$

and then $B \neq 0$. Let us study the function

$$
f(c)=\cos c-(\theta-c-1) \sin c, \quad 0 \leq c \leq \theta .
$$

We have $f(0)=1>0$ and $f(\theta)=\cos \theta+\sin \theta$, which is nonpositive for $\theta \geq \frac{3}{4} \pi$. Thus, by the intermediate value theorem, there is a point coupled to 0 for $\theta>\frac{3}{4} \pi$. Therefore Theorems 2.1 and 2.2 provide the same information, namely, that there exists no weak minimum for $\theta>\frac{3}{4} \pi$. 
Let us prove now that the functional is indeed nonnegative for $\theta \in\left[0, \frac{3}{4} \pi\right]$ and achieves, therefore, its minimum at $x(\cdot)=0$.

Assume $\theta<\frac{3}{4} \pi$. Let $w(t)=\tan \left(t-\theta+\frac{1}{4} \pi\right)$ on $[0, \theta] ; w(\cdot)$ is continuous on this interval and, for an admissible $x(\cdot)$, we have

$$
\begin{aligned}
J(x ; \theta) & =J(x ; \theta)+\frac{1}{2} \int_{0}^{\theta} \frac{d}{d s}\left\{\tan \left(s-\theta+\frac{1}{4} \pi\right) x^{2}(s)\right\} d s-\frac{1}{2} x^{2}(\theta) \\
& =\frac{1}{2} \int_{0}^{\theta}\left\{\dot{x}(s)+\tan \left(s-\theta+\frac{1}{4} \pi\right) x(s)\right\}^{2} d s \geq 0 .
\end{aligned}
$$

For $\theta=\frac{3}{4} \pi$, the term we added contains an improper integral. Assume, for the moment, that $x(\cdot) \in C^{1}$, and use $x(0)=0$ to obtain

$$
\begin{aligned}
\frac{1}{2} \int_{0}^{\frac{3}{4} \pi} \frac{d}{d s} & \left\{\tan \left(s-\frac{1}{2} \pi\right) x^{2}(s)\right\} d s \\
& =\frac{1}{2} x^{2}\left(\frac{3}{4} \pi\right)-\lim _{s \rightarrow 0^{+}} \tan \left(s-\frac{1}{2} \pi\right) x^{2}(s)=\frac{1}{2} x^{2}\left(\frac{3}{4} \pi\right) .
\end{aligned}
$$

Hence the improper integral is finite and inequality (2.3) holds even when $\theta=$ $\frac{3}{4} \pi, x(\cdot) \in C^{1}$. But $C^{1}$ is known to be dense in $H^{1,2}$ and thus the result follows.

It could happen that, subject to $(1.4), J_{2}(\eta)$ is indefinite but there are no coupled points with $b$ in $(a, b)$. This phenomenon mainly occurs because there could be nonzero constant functions admissible for the accessory problem. In order to study the behaviour of the functional (1.3) along such functions we will use the following notion, similar to the one in [10].

Definition 2.3. The problem (BVP) is said to be regular if

$$
\left(\alpha^{T}, \alpha^{T}\right) \Gamma\left(\begin{array}{c}
\alpha \\
\alpha
\end{array}\right)+\alpha^{T} \int_{a}^{b} P(s) d s \alpha \geq 0 \quad \forall \alpha \in \mathbf{R}^{n}: D\left(\begin{array}{c}
\alpha \\
\alpha
\end{array}\right)=0 .
$$

The second order necessary condition mentioned before in terms of (1.3)(1.4) yields the next result.

Theorem 2.3. If $\hat{x}(\cdot)$ is a weak local minimum for (1.1) and (1.2), then the problem $(B V P)$ is regular.

Although the result given by Theorem 2.3 is trivial, it plays a significant role, which is to complete the set of second order necessary conditions for weak local minimum. This is illustrated by Example 2 presented in the next section.

In order to prove Theorems 2.1 and 2.2 we introduce the following augmented problem, which is denoted by $(\mathrm{ABVP})^{*}$ :

Minimize

$$
\begin{aligned}
\widetilde{J}_{2}(c, \eta):= & \frac{1}{2}\left(\eta^{T}(c), \eta^{T}(b)\right) \Gamma\left(\begin{array}{l}
\eta(c) \\
\eta(b)
\end{array}\right)+\frac{1}{2} \eta^{T}(c) \int_{a}^{c} P(s) d s \eta(c) \\
& +\frac{1}{2} \int_{c}^{b}\left\{\eta^{T}(s) P(s) \eta(s)+2 \dot{\eta}^{T}(s) Q(s) \eta(s)+\dot{\eta}^{T}(s) R(s) \dot{\eta}(s)\right\} d s,
\end{aligned}
$$


over all absolutely continuous $\eta(\cdot)$, which satisfy the boundary condition

$$
D\left(\begin{array}{l}
\eta(c) \\
\eta(b)
\end{array}\right)=0 \text {. }
$$

Since $\widetilde{J}_{2}(a ; \eta)=J_{2}(\eta)$ the problem (ABVP) ${ }^{*}$ can be seen as restriction to the subinterval $[c, b]$ of (BVP) ${ }^{*}$. Furthermore, (ABVP) ${ }^{*}$ preserves the nonnegativity of the functional as shown by the following

Lemma 1. If $J_{2}(\eta) \geq 0$ for all $\eta(\cdot) \in A C$ satisfying (1.4) then $\forall c \in[a, b]$, $\widetilde{J}_{2}(c ; \eta) \geq 0$ for all $\eta(\cdot) \in A C$ satisfying $(2.5)$.

Proof. Let $c \in[a, b)$, and let $\eta(\cdot) \in A C$ satisfy (2.5). If $c=a$, the result is trivial. Take $c \in(a, b)$ and define

$$
\bar{\eta}(t)= \begin{cases}\eta(t) & \text { on }[c, b], \\ \eta(c) & \text { on }[a, c) .\end{cases}
$$

Since $D\left(\begin{array}{l}\eta(c) \\ \eta(b)\end{array}\right)=0$, the function $\bar{\eta}(\cdot)$ is admissible for $J_{2}(\cdot)$, and hence $J_{2}(\bar{\eta}) \geq$ 0 . That is,

$$
\begin{aligned}
J_{2}(\bar{\eta})= & \frac{1}{2}\left(\eta^{T}(c), \eta^{T}(b)\right) \Gamma\left(\begin{array}{l}
\eta(c) \\
\eta(b)
\end{array}\right)+\frac{1}{2} \eta^{T}(c) \int_{a}^{c} P(s) d s \eta(c) \\
& +\frac{1}{2} \int_{c}^{b}\left\{\eta^{T}(s) P(s) \eta(s)+2 \dot{\eta}^{T}(s) Q(s) \eta(s)+\dot{\eta}^{T}(s) R(s) \dot{\eta}(s)\right\} d s \geq 0,
\end{aligned}
$$

which shows that $\widetilde{J}_{2}(c ; \eta) \geq 0$.

For $c=b$, the required result becomes

$$
\widetilde{J}_{2}(b ; \eta)=\frac{1}{2}\left(\eta^{T}(b), \eta^{T}(b)\right) \Gamma\left(\begin{array}{l}
\eta(b) \\
\eta(b)
\end{array}\right)+\frac{1}{2} \eta^{T}(b) \int_{a}^{b} P(s) d s \eta(b) \geq 0,
$$

for all $\eta(b) \in \mathbf{R}^{n}$ that satisfy $D\left(\begin{array}{l}\eta(b) \\ \eta(b)\end{array}\right)=0$. The latter is the regularity condition which is satisfied due to Theorem 2.3.

The next results say that if the (ABVP) ${ }^{*}$ has a minimum, then the optimal arcs are completely characterized by the first order necessary conditions.

Lemma 2. Let $\tilde{J}_{2}(c ; \eta) \geq 0$ for some $c \in[a, b]$ and for all $\eta(\cdot) \in A C$ satisfying (2.5). Then $\tilde{J}_{2}(c ; \eta)=0$ if and only if

(i) there exist $\xi(\cdot) \in A C$ such that $(\eta(\cdot), \xi(\cdot))$ satisfies the Jacobi system (1.7) on $[c, b]$,

$$
D\left(\begin{array}{c}
\eta(c) \\
\eta(b)
\end{array}\right)=0, \quad\left(\begin{array}{c}
\xi(c) \\
-\xi(b)
\end{array}\right)=\Gamma\left(\begin{array}{c}
\eta(c) \\
\eta(b)
\end{array}\right)+D^{T} \lambda+\left(\begin{array}{c}
\int_{a}^{c} P(s) s d \eta(c) \\
0
\end{array}\right)
$$

for some $\lambda \in \mathbf{R}^{k}$.

Proof. Necessity. If $\eta(\cdot) \in A C$ satisfying $(2.5)$ is such that $\widetilde{J}_{2}(c ; \eta(\cdot))=0$ then, since the problem is quadratic and the strengthened Legendre condition holds, 
$\eta(\cdot)$ satisfies the Euler-Lagrange equation and the corresponding transversality conditions [3, p. 45, Example 2] which are exactly (i) and (ii).

Sufficiency. Let $(\eta(\cdot), \xi(\cdot))$ be a solution on $[c, b]$ of (1.7) satisfying (ii). Then it can be easily verified that

$$
\begin{aligned}
\widetilde{J}_{2}(c ; \eta)= & \frac{1}{2}\left(\eta^{T}(c), \eta^{T}(b)\right) \Gamma\left(\begin{array}{l}
\eta(c) \\
\eta(b)
\end{array}\right)+\frac{1}{2} \int_{c}^{b} \frac{d}{d t}\left(\xi^{T}(s) \eta(s)\right) d s \\
& +\frac{1}{2} \eta^{T}(c) \int_{a}^{c} P(s) d s \eta(c)=0 .
\end{aligned}
$$

Remark 2.2. In the previous lemma, condition (ii) easily implies

(iii)

$$
\begin{gathered}
D\left(\begin{array}{l}
\eta(c) \\
\eta(b)
\end{array}\right)=0 \\
\left(\begin{array}{l}
\eta(c) \\
\eta(b)
\end{array}\right)^{T}\left[\left(\begin{array}{l}
-\xi(c) \\
+\xi(b)
\end{array}\right)+\Gamma\left(\begin{array}{l}
\eta(c) \\
\eta(b)
\end{array}\right)+\left(\begin{array}{c}
\int_{a}^{c} P(s) d s \eta(c) \\
0
\end{array}\right)\right]=0 .
\end{gathered}
$$

On the other hand, from the above proof, conditions (i) and (iii) yield that $\widetilde{J}_{2}(c, \eta)=0$. Hence, by the first-order necessary conditions, there exists $\bar{\xi}$ such that $(\eta, \bar{\xi})$ satisfy $(1.7)$ on $[c, b]$ and condition (ii) of Lemma 2. Since $B(\cdot)$ is invertible it follows that $\bar{\xi}=\xi$. Thus, under the conditions of Lemma 2 , conditions (ii) and (iii) are equivalent.

By Lemma 2 , whenever $\widetilde{J}_{2}(c ; \eta)$ attains its mimimum at $\eta(\cdot)$, we can extend this function to $[a, b]$ by extending the corresponding solution $(\eta(\cdot), \xi(\cdot))$ of system (1.7). The result below states that this extension to $[a, b]$ has to be constant on $[a, c]$. In the classical case, that is, when we have fixed endpoints, $\eta(c)$ is then zero and therefore, $(\eta(\cdot), \xi(\cdot))$ is identically zero on $[a, c]$ and satisfies trivially (1.7).

Lemma 3. Assume that $\widetilde{J}_{2}(c ; \eta) \geq 0$ for all $c \in[a, b]$ and for all $\eta(\cdot) \in A C$ satisfying (2.5). Suppose that, for some $c \in(a, b), \widetilde{J}_{2}(c ; \cdot)$ has a nonzero minimizer $\eta(\cdot)$. Then its extension satisfies

$$
\eta(\cdot) \equiv \eta(c) \text { on }[a, c] .
$$

Proof. Let $(\eta(\cdot), \xi(\cdot))$ denote the extension to $[a, b]$ of the solution of system (1.7) corresponding to $\eta(\cdot)$. Since

$$
D\left(\begin{array}{l}
\eta(c) \\
\eta(b)
\end{array}\right)=0
$$

the function

$$
\bar{\eta}(t)= \begin{cases}\eta(t) & \text { on }[c, b], \\ \eta(c) & \text { on }[a, c),\end{cases}
$$

is admissible for (BVP) ${ }^{*}$ and $J_{2}(\bar{\eta}(\cdot))=\widetilde{J}_{2}(c ; \eta(\cdot))=0$. Thus $\bar{\eta}(\cdot)$ is optimal for (BVP) ${ }^{*}$ and, therefore, there exist $\bar{\lambda}, \bar{\xi}(\cdot)$, such that $(\bar{\eta}(\cdot), \bar{\xi}(\cdot))$ satisfies the 
Jacobi system (1.7) and the corresponding transversality conditions. Since $\hat{x}(\cdot)$ is $C^{1}$, the coefficients of the system (1.7), and hence $\dot{\bar{\eta}}(\cdot)$, are continuous. But

$$
\lim _{t \rightarrow c^{-}} \dot{\bar{\eta}}(t)=0
$$

and then also

$$
\lim _{t \rightarrow c^{+}} \dot{\bar{\eta}}(t)=0=\dot{\bar{\eta}}(c) .
$$

From (1.6) we obtain $\bar{\xi}(c)=\xi(c)$. By the uniqueness theorem for ordinary differential equations we have

$$
(\eta(\cdot), \xi(\cdot)) \equiv(\bar{\eta}(\cdot), \bar{\xi}(\cdot)) \quad \text { on }[a, b]
$$

and hence $\eta(\cdot) \equiv \eta(c)$ on $[a, c]$.

Proof of Theorem 2.1. If $\hat{x}(\cdot)$ is a weak local minimum for (BVP) then $J_{2}(\eta) \geq$ 0 for all $\eta(\cdot)$ satisfying (1.4). Suppose there exists $c_{0} \in(a, b)$ coupled with $b$ with corresponding $\left(\eta_{0}(\cdot), \xi_{0}(\cdot)\right)$ and $\lambda_{0}$. By Lemma $1, \widetilde{J}_{2}(c ; \eta) \geq 0$ for all $\eta(\cdot)$ such that $D\left(\begin{array}{l}\eta(c) \\ \eta(b)\end{array}\right)=0$, hence from Lemma 2 and the boundary conditions we get $\widetilde{J}_{2}\left(c_{0} ; \eta_{0}\right)=0$. Finally, using Lemma 3 , we obtain $\eta_{0}(\cdot) \equiv \eta(c)$ on $[a, c]$, which contradicts Definition 2.1.

The proof of Theorem 2.2 is analogous.

Remark 2.3. It is clear that, if we replace in Definition 2.1 the boundary conditions by condition (iii) of Remark 2.2, we obtain a more general concept of a coupled point. But, from the same remark, it follows that Theorem 2.1 is equivalent to the result that could be obtained using this new concept.

\section{APPLICATIONS TO PERIODIC PROBLEMS}

In this section we apply the previous results to the study of a periodic problem in the calculus of variations, namely:

Minimize

$$
J(x):=\gamma(x(T))+\int_{0}^{T} L(x(s), \dot{x}(s)) d s,
$$

over all absolutely continuous $x(\cdot)$, subject to the periodic boundary condition:

$$
x(0)=x(T) .
$$

We will impose the same assumptions about the lagrangian as in the previous sections. If the lagrangian is time dependent one needs to suppose that it is $T$-periodic, that is, $L(0, \cdot, \cdot)=L(T, \cdot, \cdot)$.

Assume, moreover, that $\hat{x}(\cdot)$ is a weak local minimum that satisfies the EulerLagrange equation and the corresponding transversality conditions, i.e.,

$$
\frac{d}{d t} \widehat{L}_{v}(t)=\widehat{L}_{x}(t)
$$


and

$$
\widehat{L}_{v}(0)=\widehat{L}_{v}(T)+\nabla \gamma(x(T)) .
$$

Remark 3.1. Note that a minimizer may not satisfy the Euler-Lagrange equation, as some examples in [1] and [4] show. This does not happen if the minimizer is Lipschitz continuous (see Theorem 2.6 in [3, p. 58]).

In the previous part of the paper we have assumed the optimal solution of (BVP) to be $C^{1}$. Here, we recall a result by $\mathrm{L}$. Tonelli which provides assumptions on the data that insure the smoothness of the optimal solution:

Theorem 3.1 (L. Tonelli; see e.g. [3]). Let $L(x, v)$ be $C^{1}$ on $\Omega \times \mathbf{R}^{n}$, where $\Omega$ is a closed subset of $\mathbf{R}^{n}$. Suppose that $\hat{x}(t) \in \Omega$ for all $t \in[0, T]$ and

$$
\forall t \in[0, T], L_{v}(\hat{x}(t), u) \neq L_{v}(\hat{x}(t), v) \text { for all } u, v \in \mathbf{R}^{n}, u \neq v .
$$

If $\hat{x}(\cdot)$ is a solution of (3.3) that satisfies (3.4), then it is $C^{1}$.

From now on we will assume that the conditions of the preceding theorem are satisfied and hence $\hat{x}(\cdot)$ is $C^{1}$.

Remark 3.2. Note that, in general, it is not possible to extend $\hat{x}(\cdot)$ to $\mathbf{R}$ while preserving smoothness. However, if $\gamma(\cdot) \equiv 0, \hat{x}(\cdot)$ satisfies (3.3) and (3.4), and relation (i) of Theorem 3.1 holds, then (3.4), that is,

$$
L_{v}(\hat{x}(0), \dot{\hat{x}}(0))=L_{v}(\hat{x}(T), \dot{\hat{x}}(T)) \text {, }
$$

and the periodicity boundary conditions on $\hat{x}(\cdot)$ imply $\dot{\hat{x}}(0)=\dot{\hat{x}}(T)$, and thus $\hat{x}(\cdot)$ can be extended as a $C^{1} T$-periodic function to $\mathbf{R}$.

Taking into account the special form of the boundary conditions, the accessory problem (BVP) ${ }^{*}$ becomes:

Minimize

$$
\begin{aligned}
J_{2}(\eta):= & \frac{1}{2} \eta^{T}(T) \Gamma \eta(T) \\
& +\frac{1}{2} \int_{0}^{T}\left\{\eta^{T}(s) \widehat{L}_{x x}(s) \eta(s)+2 \dot{\eta}^{T}(s) \widehat{L}_{v x}(s) \eta(s)+\dot{\eta}^{T}(s) \widehat{L}_{v v}(s) \dot{\eta}(s)\right\} d s
\end{aligned}
$$

over all absolutely continuous $\eta(\cdot)$, subject to the boundary conditions: $\eta(0)=$ $\eta(T)$, where $\Gamma:=\nabla^{2} \gamma(\hat{x}(a), \hat{x}(b))$.

Remark 3.3. Under the assumptions of the preceding remark, it follows that the coefficients of the accessory problem are $T$-periodic.

Using the same notations as in $\S 2$, an extremal for the accessory problem will be a pair $(\eta(\cdot), \xi(\cdot))$ of absolutely continuous functions that satisfies the Jacobi system (1.7) and the boundary conditions

$$
\eta(0)=\eta(T), \quad \xi(0)=\xi(T)+\Gamma \eta(T) .
$$

Once again, since the coefficients of the Jacobi system are continuous, the functions $\eta(\cdot), \xi(\cdot)$ are $C^{1}$ and, as in Remark 3.2, they can be extended smoothly to $\mathbf{R}$ if $\gamma(\cdot) \equiv 0$.

The next result is a direct application to the problem (3.1)-(3.2) of the results obtained in the previous sections. 
Corollary 3.1. Suppose that $\hat{x}(\cdot)$ is a weak local minimum for (3.1)-(3.2). Then the following conditions are satisfied:

(i) for all $\alpha \in \mathbf{R}^{n}$

$$
\alpha^{T}\left[\Gamma+\int_{0}^{T} P(s) d s\right] \alpha \geq 0,
$$

that is, the problem is regular, and

(ii) there exists no nonzero $(\eta(\cdot), \xi(\cdot))$ solution of the Jacobi system (1.7) such that for some $c \in(0, T)$, the following coupling conditions are satisfied:

$$
\eta(\cdot) \not \equiv \eta(c) \text { on }[a, c], \quad \eta(c)=\eta(T),
$$

and

$$
\xi(c)=\xi(T)+\Gamma \eta(T)+\int_{a}^{c} P(s) d s \eta(T) .
$$

That is, there is no point $c \in(0, T)$ coupled with $T . A$ similar statement holds true for points coupled with zero.

We end this section by analyzing the functional studied in Example 1, but with periodic boundary conditions. This functional has been often used to test the necessary conditions. The two examples presented here complete a survey of possible boundary conditions. The case of fixed endpoints is analyzed e.g. in [3], and that of free endpoints is studied in [10].

Example 2. Minimize

$$
J(x ; T)=\frac{1}{2} x^{2}(T)+\frac{1}{2} \int_{0}^{T}\left\{\dot{x}^{2}(s)-x^{2}(s)\right\} d s
$$

over all absolutely continuous $x(\cdot)$, subject to the periodic boundary conditions $x(0)=x(T)$.

Let us first check the regularity condition. For $x(\cdot) \equiv k$,

$$
J(k ; T)=\frac{1}{2} k^{2}(1-T) \geq 0
$$

for $0 \leq T \leq 1$.

Once again the Jacobi system of (3.5) is the harmonic oscillator, whose solutions are of the form $\eta(t)=A \cos t+B \sin t, \xi(t)=-A \sin t+B \cos t$. The point $c \in(0, T)$ is coupled with 0 if

that is,

$$
\eta(0)=\eta(c), \quad \xi(0)=\xi(c)+\eta(0)+\int_{c}^{T} P(s) d s \eta(0)
$$

$$
A \cos c+B \sin c=A, \quad B \cos c-A \sin c=B-A(1+c-T) .
$$

Let us find the solutions of the above system. Solve the first with respect to $\sin c$ and substitute in the second, to obtain

$$
\begin{aligned}
& \sin c=\frac{A^{2}}{A^{2}+B^{2}}[1+c-T], \\
& \cos c=1-\frac{A B}{A^{2}+B^{2}}[1+c-T] .
\end{aligned}
$$


Since we can easily verify that $A \neq 0$, we can set $\beta:=\frac{B}{A}$ and use the relation $\cos ^{2} c+\sin ^{2} c=1$ to deduce from the above system:

$$
[1+c-T][1+c-T-2 \beta]=0 .
$$

Since $T \leq 1$ and $c>0$, it follows that $1+c-T>0$, and hence, $c=2 \beta+T-1$. By substituting this value in the first equation of (3.6), we obtain

$$
F(\beta):=\sin (2 \beta+T-1)-\frac{2 \beta}{1+\beta^{2}}=0 .
$$

Let us study this function for $c \in[0, T]$, that is, for $0 \leq T-1+2 \beta \leq T$ or equivalently $0 \leq k_{0}:=(1-T) / 2 \leq \beta \leq \frac{1}{2}$. Compute

$$
F\left(\frac{1}{2}\right)=\sin T-\frac{4}{5} \geq 0 \text { for } T \geq \bar{T}, \quad \text { where } \bar{T}=\sin ^{-1} \frac{4}{5}
$$

and

$$
F\left(k_{0}\right)=-\frac{2 k_{0}}{1+k_{0}^{2}} \leq 0 .
$$

Hence, if $T>\bar{T}$, there is a point $c \in(0, T)$ coupled with zero.

Let us show now that $J_{2}(x ; T) \geq 0$ for all $T \in[0, \bar{T}]$. In fact, $J_{2}(x ; T)>0$ for $T \in(0, \bar{T})$ and $J_{2}(x ; T) \geq 0$ for $T=0$ or $T=\bar{T}$. Let $w(t)=\tan \left(t-\frac{T}{2}\right)$ for $T \in[0, \bar{T}] . w(\cdot)$ is continuous and

$$
\begin{aligned}
J_{2}(x ; T)= & \frac{1}{2} x^{2}(T)+\frac{1}{2} \int_{0}^{T}\left\{\dot{x}^{2}(s)-x^{2}(s)\right\} d s \\
& +\frac{1}{2} \int_{0}^{T} \frac{d}{d s}\left\{\tan \left(s-\frac{T}{2}\right) x^{2}(s)\right\} d s \\
& -\frac{1}{2} \tan \left(\frac{T}{2}\right) x^{2}(T)+\frac{1}{2} \tan \left(-\frac{T}{2}\right) x^{2}(0) \\
= & \frac{1}{2}\left[1-2 \tan \frac{T}{2}\right] x^{2}(T)+\frac{1}{2} \int_{0}^{T}\left\{\dot{x}(s)+\tan \left(s-\frac{T}{2}\right)\right\}^{2} d s
\end{aligned}
$$

Since one can show that $\tan \frac{T}{2} \leq \frac{1}{2}$ for $T \leq \bar{T}$ the conclusion follows.

Concluding remarks. The development of sufficient conditions in terms of coupled points is the subject of further research in order to render this theory complete.

A different approach to this problem was initiated in the 30's by Morse (for detailed exposition and references see [6]). Also, sufficient conditions in terms of Riccati equations are provided by Reid in [7]. The relation between these types of results and the ones given here need to be investigated.

Acknowledgment. We wish to thank the referee whose comments greatly helped to improve the exposition of the paper. 


\section{REFERENCES}

1. J. M. Ball and V. J. Mizel, One-dimensional variational problems whose minimizers do not satisfy the Euler-Lagrange equation, Arch. Rational Mech. Anal. 90 (1985), 325-388.

2. G. A. Bliss, Lectures on the calculus of variations, Univ. of Chicago Press, Chicago, 1946.

3. L. Cesari, Optimization-Theory and applications, Springer-Verlag, New York, 1983.

4. F. H. Clarke and R. B. Vinter, Regularity properties of the solutions to the basic problem in the calculus of variations, Trans. Amer. Math. Soc. 289 (1985), 73-98.

5. M. R. Hestenes, Calculus of variations, Wiley, New York, 1969.

6. M. Morse, Variational analysis, Wiley, New York, 1973.

7. W. T. Reid, Riccati differential equations, Academic Press, New York, 1972.

8. H. Sagan, Introduction to the calculus of variations, McGraw-Hill, New York, 1969.

9. J. L. Speyer and R. T. Evans, A second variational theory for optimal periodic processes, IEEE Trans. Automat. Control AC-29 (1984), 138-148.

10. V. Zeidan and P. Zezza, An extension of the conjugate point conditions to the case of variable endpoints, Proc. 27th IEEE Conf. on Decision and Control, 1988, pp. 1187-1191.

11. V. Zeidan and P. Zezza, Variable end point problems in the calculus of variations: Coupled Points, Proc. Eighth Internat. Conf. on Analysis and Optimization of Systems, 1988, pp. 372380.

Department of Applied Mathematics, University of Waterloo, Waterloo, Ontario, N2L 3G1, CANADA

Dipartimento di Sistemi e Informatica, Via di S. Marta 3, 50139 Firenze, Italy

E-mail (Vera Zeidan): VMZEIDAN @ BITNET

E-mail (PierLuigi Zezza): EQUADIFF @ IFIIDG 